\title{
Propiedades del suelo en bosques quemados de Austrocedrus chilensis en Patagonia, Argentina
}

\author{
Soil characteristics in burned Austrocedrus chilensis forests in Patagonia, Argentina \\ María Florencia Urretavizcaya
}

Centro de Investigación y Extensión Forestal Andino Patagónico (CIEFAP), CC 14, 9200 Esquel,

Chubut, Argentina, tel./fax: 54-2945-453948/450175, mfurretavizcaya@ciefap.org.ar

\begin{abstract}
SUMMARY
Austrocedrus chilensis is the most important natural conifer in the Patagonian Andes region of southern Argentina where there are 141,000 hectares of mainly pure stands. Fire is the main disturbance in the dynamics of these forests. The objective of the study was to analyze possible differences in chemical, physical and biological soil properties among unburned (NQ), partially burned

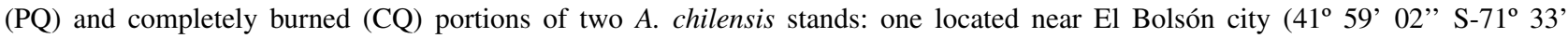

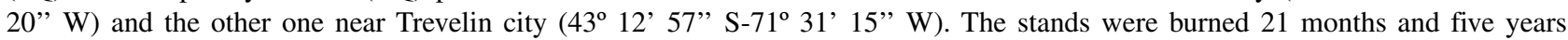
before running the studies respectively. The following soil properties expected to influence forest recovery were measured: $\mathrm{pH}$, electric conductivity (EC), organic carbon $(\mathrm{C})$, total nitrogen $(\mathrm{N})$, interchangeable potassium $(\mathrm{K})$, calcium $(\mathrm{Ca})$, magnesium $(\mathrm{Mg})$, and nitrogen in the microorganisms $(\mathrm{N}-\mathrm{BM})$ in the upper $10 \mathrm{~cm}$ of soil. At El Bolsón study the PQ and CQ portions of the stand had higher $\mathrm{pH}$ and EC and less $\mathrm{C}, \mathrm{N}, \mathrm{Mg}$ and $\mathrm{N}-\mathrm{BM}$ than the NQ portion. These changes may be related to the combustion of the organic matter. These differences were not registered at the Trevelin study; I speculate that at this site soil nutrient content could have been recovered because much more time elapsed between the occurrence of the fire and the installation of the study. It is desirable that after a forest fire the vegetation get reestablished as soon as possible to prevent nutrient loss and hasten the recovery of soils characteristics; this can be facilitated by planting A. chilensis.
\end{abstract}

Key words: ciprés de la cordillera, natural forest, fire effects, postfire soil conditions.

\section{RESUMEN}

Austrocedrus chilensis, ciprés de la cordillera, es la conífera nativa más importante de la región de los bosques andino patagónicos de Argentina, donde existen 141.000 ha de rodales fundamentalmente puros. El fuego es el principal disturbio de la dinámica de estos bosques. Este estudio tuvo por objetivo analizar posibles diferencias en propiedades químicas, físicas y biológicas del suelo entre lugares no quemados (NQ), parcialmente quemados (PQ) y completamente quemados (CQ) de bosques de A. chilensis.

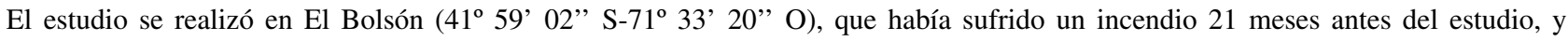
Trevelin ( $43^{\circ} 12^{\prime} 57^{\prime}$ ' S-71 $31^{\prime}$ ' 15" O), quemado casi cinco años antes. Se midieron propiedades del suelo en los primeros $10 \mathrm{~cm}$ de profundidad que podrían tener más influencia sobre la recuperación del bosque: densidad aparente, $\mathrm{pH}$, conductividad eléctrica $(\mathrm{CE})$, carbono orgánico $(\mathrm{C})$, nitrógeno total $(\mathrm{N})$, potasio intercambiable $(\mathrm{K})$, calcio $(\mathrm{Ca})$, magnesio $(\mathrm{Mg})$ y nitrógeno retenido en la biomasa microbiana (N-BM). En El Bolsón los sectores PQ y CQ presentaron mayor pH y CE y menor cantidad de C, N, Mg y $\mathrm{N}$-BM respecto al sector NQ, lo que podría estar relacionado con la combustión de la materia orgánica. En Trevelin no se detectaron esas diferencias, sugiriendo que el contenido de nutrientes del suelo se habría recuperado por haber transcurrido un tiempo mucho más prolongado desde la ocurrencia del fuego. Luego de un incendio es deseable que la vegetación se restablezca lo antes posible para mitigar las posibles pérdidas de nutrientes y favorecer la recuperación de las propiedades de suelo, lo que puede ser beneficiado mediante la plantación de A. chilensis.

Palabras clave: ciprés de la cordillera, bosque nativo andino patagónico, efectos del fuego, condiciones postfuego del suelo.

\section{INTRODUCCIÓN}

En la última década, extensos fuegos han ocurrido en la mayoría de los continentes, afectando un amplio rango de ecosistemas (Williams y Bradstock 2008) y los bosques andino patagónicos no han sido una excepción. Las consecuencias ecológicas de estos fuegos son específicas para cada sitio y altamente asociadas al modo reproductivo de las especies dominantes, así como a las condiciones climáticas y herbivoría con posterioridad al evento de fuego (Veblen et al. 2008). Para asistir a la restauración de los ecosistemas afectados y a la planificación del manejo de fuego en estos bosques nativos, es necesario avanzar en el conocimiento sobre el efecto de este disturbio sobre los distintos componentes del ecosistema y su resiliencia. 
Austrocedrus chilensis (D. Don) Pic. Ser et Bizarri se distribuye a lo largo de una franja discontinua de no más de $50 \mathrm{~km}$ de ancho. Aparece en el sector chileno de la Cordillera de Los Andes a los 32 39' de latitud sur (S) donde se encuentran sólo individuos aislados, pequeños y de troncos defectuosos (Schlegel 1962). A partir del paralelo $34^{\circ} 45^{\prime} \mathrm{S}$ aparece formando asociaciones boscosas de medianas a pequeñas superficies, asociadas con especies esclerófitas o del género Nothofagus (Donoso 1993). En la latitud $38^{\circ} \mathrm{S}$ atraviesa la Cordillera de Los Andes hacia Argentina para componer, a partir de los $39^{\circ}$ 30' S y hasta los $43^{\circ} 44^{\prime} \mathrm{S}$, los bosques más prominentes y continuos de la especie (Pastorino y Gallo 2000). En el extremo austral de su distribución en Argentina las formaciones de Trevelin y Corcovado (43 $10^{\circ}$ y $43^{\circ} 30^{\prime} \mathrm{S}$, respectivamente), atraviesan la Cordillera de Los Andes y la especie aparece nuevamente en Chile.

Aproximadamente el $80 \%$ del total de la superficie cubierta por A. chilensis (141.000 ha) se ubica en Argentina en una franja angosta entre los $37^{\circ} 08^{\prime} 09^{\prime}$ ' $\mathrm{S}$ y los $43^{\circ}$ 43' 57" S (Bran et al. 2002) en la vertiente oriental de la Cordillera de Los Andes, que luego da paso a la estepa Patagónica. Longitudinalmente, los bosques de A. chilensis en este país se ubican en un marcado gradiente de humedad que abarca desde sitios muy húmedos en el oeste a xéricos en el este, en el piedemonte de la cordillera. Austrocedrus chilensis es una especie longeva y forma rodales densos, puros o mixtos con Nothofagus dombeyi (Mirb.) Oerst. (coihue) en los sitios más húmedos, rodales puros en sitios más secos y rodales abiertos en sitios xéricos cerca del ecotono entre los bosques y la estepa patagónica (Veblen et al. 1995).

La mayor parte de los suelos de la región andino patagónica donde crece $A$. chilensis son derivados de cenizas volcánicas. La evolución de éstos está ligada a la distribución de humedad y a la existencia de barreras naturales que actuaron frenando la distribución de las cenizas (Etchevehere 1972). En los sitios de mayor precipitación, al oeste, se encuentran los andosoles, los cuales son considerados como suelos de alta fertilidad con una provisión adecuada de nutrientes (a excepción de fósforo) y alta capacidad de almacenamiento de agua (Bornemisza 1982, Colmet Dâage et al. 1993). Los dos componentes esenciales de estos suelos son materiales amorfos: la materia orgánica degradada y el alófano. Ambos forman complejos organo-minerales muy estables, independientemente de la vegetación presente (Bornemisza 1982, Colmet Dâage et al. 1995). La formación de las sustancias alofánicas requiere un medio con humedad permanente, bien drenado y aireado, con una deshidratación del perfil que rara vez se acerque al punto de marchitez permanente. En suelos sometidos a un ligero desecamiento estacional, hay tendencias a una mejor organización de las sustancias alofánicas y una ligera cristalización, apareciendo la imogolita, que es un mineral de transición entre alófanos y arcillas cristalinas (Colmet Dâage et al. 1993, 1995). La densidad aparente en conjunto con el espacio poroso, son indicadores de propiedades importantes de la estabilidad del suelo y del crecimiento de las plantas. En general los andosoles presentan baja densidad aparente debido al gran espacio poroso de los minerales alofánicos e imogolita, al alto contenido de materia orgánica y a la baja densidad de las partículas alofánicas. La materia orgánica, junto a otros agentes cementantes, juega un rol fundamental en las uniones entre partículas, conduciendo a la formación de agregados estables que retiene altos contenidos de agua.

Los bosques de A. chilensis han estado sometidos a distintos regímenes de perturbaciones como sismos, viento y herbivoría (Veblen et al. 1996), pero ha sido el fuego el disturbio más importante (Veblen et al. 1992, Kitzberger 1994). Debido a su corteza delgada, que no opone resistencia al calor que daña los tejidos de conducción, y a su escasa capacidad para reproducirse vegetativamente, los incendios intensos producen gran mortalidad (Donoso 1981). Luego de la ocurrencia de incendios, el patrón de regeneración natural de $A$. chilensis varía de acuerdo a las condiciones ambientales de los rodales (Veblen et al. 1995) $\mathrm{y}$, como sucede en otros ecosistemas, a las condiciones climáticas posteriores al evento de fuego, al tamaño del área afectada y a la intensidad del disturbio (Connell y Slatyer 1977).

El fuego produce una variedad de efectos en el suelo que dependen de su intensidad, del tipo de combustible, de las características del suelo, del clima y la topografía (Wright y Bailey 1982, Pyne 1984, Díaz-Fierros et al. 1990). Puede afectar significativamente propiedades físicas, químicas y biológicas de la capa superficial del suelo (DeBano 1990, Agee 1993, Valette et al. 1994) que son esenciales para el mantenimiento de la productividad forestal a largo plazo (Agee 1993, Neary et al. 1999, Arocena y Opio 2003). El fuego altera asimismo el ciclo de nutrientes, ya que la combustión del mantillo y la materia orgánica incrementan la disponibilidad de algunos nutrientes mientras otros son volatilizados (DeBano 1990, Agee 1993, Gillon et al. 1995, Wan et al. 2001).

En ausencia de fuego, los nutrientes contenidos en la madera muerta y en la materia orgánica del suelo son reciclados por el proceso de descomposición biológica, en ambientes donde la temperatura raramente alcanza los $38^{\circ} \mathrm{C}$ $\mathrm{y}$ hay suficiente humedad como para sostener la actividad microbiana. Bajo estas condiciones, los microorganismos del suelo descomponen la materia orgánica lentamente y a lo largo del tiempo, liberando de esa manera los nutrientes esenciales (DeBano 1990). En contraste, durante un evento de fuego, los nutrientes almacenados en el material combustible y la materia orgánica del suelo están sujetos a un calentamiento severo y sometidos a varias transformaciones irreversibles durante la combustión (Raison 1979).

La respuesta de cada nutriente a esta condición difiere y es inherente a su umbral de temperatura. Este umbral de temperatura es definido como la temperatura a la cual el nutriente es volatilizado (DeBano 1990, Agee 
1993, Guillon et al. 1995, DeBano et al. 1998). Según este umbral, los nutrientes pueden ser divididos en tres categorías generales: sensibles, moderadamente sensibles y relativamente insensibles. El nitrógeno y el azufre son considerados sensibles porque tienen umbral bajo, entre 200 y $375^{\circ} \mathrm{C}$, respectivamente. El potasio y el fósforo son moderadamente sensibles, con umbrales de $774^{\circ} \mathrm{C}$, mientras que el magnesio, calcio y manganeso son relativamente insensibles, con umbrales altos de $1.107^{\circ} \mathrm{C}, 1.484^{\circ} \mathrm{C}$ y $1.962^{\circ} \mathrm{C}$, respectivamente. Una vez que la temperatura del suelo supera los $280^{\circ} \mathrm{C}$, las reacciones exotérmicas predominan y se enciende la materia orgánica (MO, Pyne 1984). Cuando la temperatura de la MO del suelo en superficie alcanza los 500 a $600^{\circ} \mathrm{C}$, se produce la combustión incandescente. Si el oxígeno está presente, la combustión produce llama y las temperaturas se pueden incrementar de 800 a $1.500^{\circ} \mathrm{C}$ (Pyne 1984, DeBano 1990).

Durante fuegos forestales, la temperatura máxima del suelo se ubica en el rango de 200 a $300^{\circ} \mathrm{C}$, pero en combustibles pesados como troncos y ramas gruesas, es común que la superficie del suelo alcance temperaturas máximas de 500 a $700^{\circ} \mathrm{C}$ y que ocurran además temperaturas instantáneas superiores a los $1.500^{\circ} \mathrm{C}$ (Dunn y DeBano 1977). El más importante y significante efecto del fuego en bosques, matorrales y pastizales es la transferencia del calor desde la biomasa encendida hacia el suelo (DeBano et al. 1998). La cantidad y duración de esa transferencia de calor determina la severidad del impacto en las propiedades físicas del sistema suelo, sus constituyentes químicos y componentes biológicos (Neary et al. 1999, 2005).

Una rápida colonización del suelo por parte de la vegetación es un factor clave en la minimización de las pérdidas de nutrientes después del fuego. La estabilización del contenido y disponibilidad de nutrientes generalmente es el resultado del incremento de nueva vegetación en los primeros años, especialmente abundante en pastos y hierbas (Christensen 1987). La efectiva recuperación de los nutrientes disueltos disminuye la posibilidad del lavado posterior al fuego (Kutiel et al. 1990). Esta recuperación, en conjunto con la inmovilización microbiana son los principales procesos que determinan la resiliencia de un ecosistema después del fuego (Weston y Attiwill 1990, Singh 1994).

Respecto al efecto del fuego sobre los bosques de A. chilensis se ha determinado que en un plazo relativamente corto luego de su ocurrencia (de uno a seis años) hay significativos cambios en la cobertura del suelo (Urretavizcaya et al. 2006), similar a lo que sucede en otros bosques de coníferas (Trabaud et al. 1985). Se puede diferenciar una primera fase de incremento rápido durante los primeros años luego del fuego, principalmente de especies gramíneas, compuestas y rebrote de especies arbustivas, y una segunda fase de incremento lento donde crecen los arbustos. En cuanto a las posibilidades del restablecimiento temprano de A. chilensis en las áreas quemadas, las mismas están fuertemente condicionadas por la escasa o nula cantidad de semillas (Urretavizcaya y Defossé 2004), así como por las elevadas y persistentes temperaturas que alcanza el suelo en esas áreas.

El conocimiento de cómo se modifican luego de un evento de fuego las propiedades del suelo superficial de estos bosques, que podrían influir sobre la recuperación del ecosistema es aún limitado. El presente estudio tiene como objetivo analizar qué cambios se reconocen en las principales propiedades químicas, físicas y biológicas del suelo en áreas de bosque de A. chilensis quemadas cinco años atrás en relación a aquellas áreas no quemadas, así como la capacidad de recuperación postincendio de esas propiedades.

\section{MÉTODOS}

Áreas de estudio. El estudio se realizó simultáneamente en dos bosques puros de A. chilensis de la zona centro-sur de distribución de la especie en Argentina. El primer rodal, El Bolsón ( $41^{\circ} 59^{\prime} 02^{\prime \prime} \mathrm{S}, 71^{\circ} 33^{\prime} 20^{\prime \prime} \mathrm{O}$ ), se encuentra en la Reserva Forestal Loma del Medio-Río Azul, vecina a la ciudad homónima, provincia de Río Negro y fue afectado por un incendio en enero de 1999. El segundo, llamado Trevelin ( $\left.43^{\circ} 12^{\prime} 57^{\prime \prime} \mathrm{S}, 71^{\circ} 31^{\prime} 15^{\prime \prime} \mathrm{O}\right)$, próximo a la localidad del mismo nombre en la provincia del Chubut, fue afectado por fuego en diciembre de 1996.

En cada rodal de estudio se identificó en el año 2000 un área rectangular ubicada a ambos lados de la línea de fuego, o sea, el límite entre la zona quemada y la no quemada. Esta área presentaba características similares en cuanto a condiciones locales como exposición y pendiente, así como similitud en la estructura del estrato arbóreo antes de la ocurrencia del incendio. En El Bolsón el área de estudio es de $64 \mathrm{~m} \times 128 \mathrm{~m}$ y la fracción no quemada del rodal tenía 81,4 años $\pm 6,2$ años y 864 A. chilensis por hectárea con una altura dominante de $18 \mathrm{~m}$ y diámetro cuadrático medio (DCM) de 20,7 cm. El área de estudio en Trevelin es de $48 \mathrm{~m}$ x $144 \mathrm{~m}$ y la porción no quemada del rodal tenía 44,2 años $\pm 6,7$ años y 958 A. chilensis por hectárea, con una altura dominante de $13 \mathrm{~m}$ y DCM de $18,7 \mathrm{~cm}$.

Los suelos de ambas áreas son derivados de ceniza volcánica. El de El Bolsón tiene un horizonte A con textura franca y el de Trevelin presenta horizonte A con textura pseudo-limosa y discontinuidad glacifluvial (La Manna 2005). El pH en FNa indica presencia de alófano en El Bolsón (pH: 11,2) y de imogolita en Trevelin $(\mathrm{pH}$ : 9,3) (Irisarri 2000).

El clima en ambos lugares de estudio es templado, el $75 \%$ de la precipitación ocurre en otoño-invierno (entre abril y septiembre) y tanto las primaveras como los veranos (de octubre a marzo) son generalmente cálidos y secos. La temperatura y la precipitación media anual son de $9,9^{\circ} \mathrm{C}$ y $921 \mathrm{~mm}$ en El Bolsón (Bustos y Rocchi 1993) y de 8, $2^{\circ} \mathrm{C}$ y $684 \mathrm{~mm}$ en Trevelin (Arbuinés 1998). El Bolsón 
es representativo de los bosques de A. chilensis mésicos mientras que Trevelin lo es de los xéricos.

Diseño de mиestreo. En cada área de estudio se establecieron al azar 15 parcelas circulares de $200 \mathrm{~m}^{2}(15,96 \mathrm{~m}$ de diámetro), que constituyeron las unidades de muestreo, las cuales quedaron ubicadas en distintas zonas del área de estudio con tres grados de afectación por fuego (figura 1). Estas unidades experimentales se establecieron en febrero del año 2000 en ambos sitios, correspondiendo a un año desde el evento de fuego en El Bolsón y a tres años y dos meses en Trevelin. Cada uno de estos tres niveles de disturbio de fuego fue considerado como un tratamiento:

- No quemado (NQ): la vegetación no fue afectada por fuego.

- Parcialmente quemado (PQ): la vegetación fue afectada por fuego pero la baja intensidad del evento permitió la supervivencia de individuos de A. chilensis mayores a $5 \mathrm{~cm}$ de DAP.

- Completamente quemado (CQ): la vegetación fue afectada por fuego de tal manera que no hubo supervivencia de individuos de A. chilensis.

Asimismo se determinó la severidad de fuego de cada tratamiento de acuerdo a la clasificación propuesta por Mutch y Swetman (1995). El tratamiento PQ de ambos sitios se corresponde con la categoría 2 de "fuegos de severidad suave a moderada" donde la mayoría de los árboles pequeños y menos del 50\% de los árboles de los

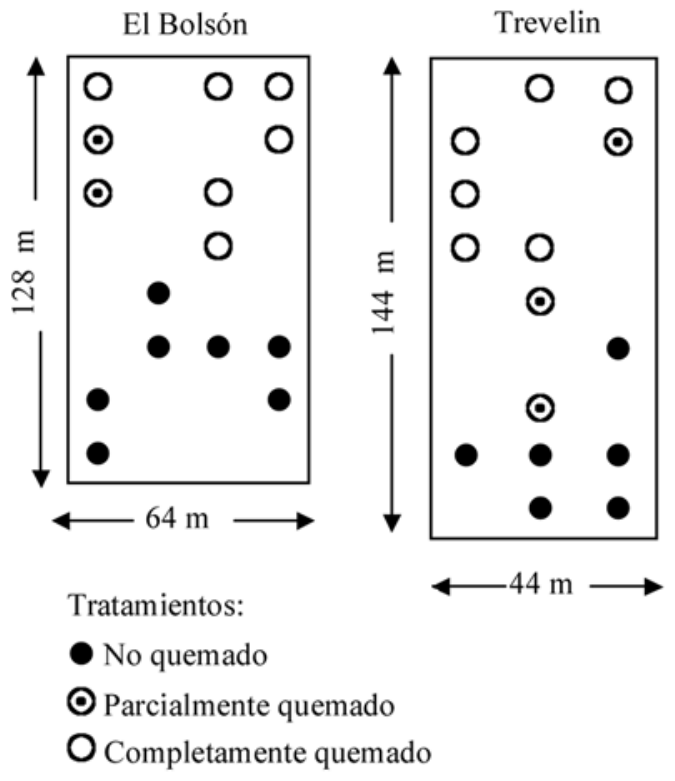

Figura 1. Distribución espacial de las unidades de muestreo circulares concéntricas $\left(200 \mathrm{~m}^{2}\right.$ y $\left.40 \mathrm{~m}^{2}\right)$ en las áreas de estudio de El Bolsón y Trevelin.

Spatial distribution of concentric circular sampling units (200 $\mathrm{m}^{2}$ and $40 \mathrm{~m}^{2}$ ) in the study areas of El Bolsón and Trevelin. estratos intermedios están muertos, ocurre coronación de algunos árboles, carbonización de la corteza de árboles vivos y parte del suelo mineral queda expuesto. El tratamiento CQ lo hace con la categoría 4 de "fuegos de alta severidad" caracterizados porque la mayoría de los árboles del estrato intermedio están muertos; más del 50\% de los árboles del estrato superior están muertos, hay consumo del follaje o parte de él, se observan cambios de color en algunos sectores del suelo, cenizas de color blanco.

Al momento de instalar las unidades de muestreo no se registró regeneración de A. chilensis en el tratamiento CQ de ninguno de los rodales de estudio. En El Bolsón, al año del incendio, la cobertura del sotobosque, principalmente de especies gramíneas y herbáceas, no fue diferente entre los tratamientos y varió de 35 a 42\%. En Trevelin la cobertura del sotobosque, especialmente de gramíneas y de arbustivas rebrotadas, fue distinta entre los tratamientos registrándose mayor cobertura en relación directa con el grado de afectación por fuego $(\mathrm{CQ}=85 \%>\mathrm{PQ}=38 \%>\mathrm{NQ}=8 \%)$ (Urretavizcaya et al. 2006).

Propiedades del suelo. En octubre del año 2001, a 21 meses del evento de fuego en El Bolsón y a casi cinco años en Trevelin, se efectuó la toma de muestras para analizar las propiedades de suelo. La misma se realizó en unidades de muestreo de $40 \mathrm{~m}^{2}$ ubicadas concéntricamente con las unidades de muestreo de $200 \mathrm{~m}^{2}$ indicadas anteriormente. Se analizó densidad aparente (DA) como propiedad física, $\mathrm{pH}$ en agua ( $\mathrm{pHa}$ ), conductividad eléctrica (CE), carbono orgánico $(\mathrm{Corg})$, nitrógeno total $(\mathrm{N})$, fósforo $(\mathrm{P})$, potasio $(\mathrm{K})$, calcio $(\mathrm{Ca})$ y magnesio $(\mathrm{Mg})$ como propiedades químicas y nitrógeno retenido en biomasa microbiana (N-BM) como propiedad biológica, según los métodos que se describen a continuación, en los primeros $10 \mathrm{~cm}$ de profundidad del suelo de ambas áreas de estudio.

Para densidad aparente se tomó una muestra al azar por parcela con un cilindro de densidad de $100 \mathrm{~cm}^{3}$. Las muestras se secaron en estufa a $105^{\circ} \mathrm{C}$ hasta peso constante, calculando luego el peso por $\mathrm{cm}^{3}$. Para las restantes propiedades se elaboró una muestra compuesta por parcela. Los análisis se realizaron en el Laboratorio de Suelos del Centro Regional Universitario Bariloche de la Universidad Nacional del Comahue. Se utilizaron muestras secas al aire y tamizadas por malla de $2 \mathrm{~mm}$ para determinar, según la metodología descrita por Sparks et al. (1996): pH en agua (suelo:agua 1:2,5); conductividad eléctrica (1:5); cationes intercambiables $(\mathrm{Ca}, \mathrm{Mg}$ y $\mathrm{K}$ ) extraídos con acetato de amonio $1 \mathrm{~N}$, seguidos de cuantificación por espectrometría de absorción atómica. En muestras tamizadas por malla de $0,5 \mathrm{~mm}$, se analizaron carbono orgánico por digestión ácida según el método de Walkley y Black (1934) y nitrógeno total por semimicro Kjeldahl (Page et al. 1982). El fósforo extraíble se determinó con bicarbonato de sodio $0,5 \mathrm{M}(\mathrm{pH}$ 8,4 , relación suelo seco: solución de 1:20) y se determinó por espectrofotometría por el método del ácido ascórbicomolibdato (Murphy y Riley 1962). El nitrógeno retenido en 
biomasa microbiana (N-BM) se determinó en las mismas muestras utilizadas para el ensayo anterior, utilizando una modificación del método de fumigación-incubación (Vitousek y Matson 1985). Las muestras se fumigaron con cloroformo líquido durante 20-24 h, posteriormente se removió el cloroformo con bomba de vacío y se incubaron durante 10 días a humedad y temperatura óptimas, y luego se extrajo $\mathrm{NH}_{4}^{+}$en $\mathrm{KCl} 2 \mathrm{M}$ (suelo: solución, 1:5) y se determinó colorimétricamente por la reacción de Berthelot (Sparks et al. 1996).

Análisis de datos. Los datos fueros analizados para cada lugar considerando los tres tratamientos (grado de afectación por fuego) con replicaciones (Trevelin: NQ $\mathrm{n}=6$, PC $\mathrm{n}=3$, CQ $\mathrm{n}=6$; El Bolsón: $\mathrm{NQ} \mathrm{n}=6$, $\mathrm{PQ} \mathrm{n}=2$, CQ $n=7)$ mediante análisis de la varianza de una vía (ANDEVA), asumiendo que el error de muestreo representa el error experimental. Esta técnica es muy empleada en trabajos de investigación ecológica aplicada (Thurow et al. 1988, Baker y Guthery 1990, Dormaar et al. 1990, Wester 1992), dado que en este tipo de estudios las unidades de muestreo no constituyen verdaderas repeticiones debido a su falta de independencia espacial (Hurlbert 1984).

Previo al ANDEVA se examinaron los supuestos de homogeneidad de la varianza y de normalidad. El primero se analizó mediante la prueba de Levenne y la observación de los residuales, y el segundo mediante la prueba de Shapiro Wilk analizando también el diagrama de normalidad (Sokal y Rohlf 1981). En los casos en que se observó heterocedasticidad, se probaron tres tipos de transformaciones: inversa, raíz cuadrada y logaritmo de la variable de respuesta, seleccionando la que presentó mejor estabilización de la varianza (SAS 1999). En El Bolsón se aplicó transformación inversa para el fósforo y el calcio, logaritmo para la conductividad eléctrica y el potasio, y raíz cuadrada para la concentración de nitrógeno total y el nitrógeno retenido en biomasa microbiana. En Trevelin se aplicó transformación inversa para la concentración de carbono orgánico y de nitrógeno total, y transformación logarítmica para el fósforo y el magnesio. En cada uno de los análisis, y para determinar entre qué tratamientos hubo diferencias significativas, se utilizó la prueba de Tukey-Kramer, que es una modificación de la prueba de Tukey para diseños desbalanceados (SAS 1999).

\section{RESULTADOS}

Las propiedades físicas y químicas de los suelos (cuadro 1) de El Bolsón y Trevelin en la zona no afectada por fuego son características de suelos derivados de cenizas volcánicas y presentan valores similares a otros suelos de la región (Buamscha et al. 1998, La Manna 2005). El Bolsón muestra semejanza con los suelos ubicados hacia el oeste, en áreas de mayor precipitación mientras que Trevelin muestra semejanza con los suelos ándicos que están ubicados hacia el este, en la transición hacia la estepa (Swindale 1969, López et al. 1992, Colmet Dâage et al. 1993).

En El Bolsón se determinó un aumento $(P<0,001)$ de $\mathrm{pHa}$ y $\mathrm{CE}$ en los tratamientos quemados (PQ y CQ) respecto al no quemado (NQ), y una disminución $(P<0,05)$ de carbono, nitrógeno, magnesio y N-BM en los mismos

Cuadro 1. Valores medios \pm error estándar de las propiedades de suelo en El Bolsón y Trevelin por tratamiento.

Mean and standard error per treatment of the soil variables measured for the study at both locations: El Bolsón and Trevelin.

\begin{tabular}{|c|c|c|c|c|c|c|c|c|}
\hline \multirow{3}{*}{ Propiedades del suelo } & \multicolumn{4}{|c|}{ El Bolsón } & \multicolumn{4}{|c|}{ Trevelin } \\
\hline & NQ & PQ & CQ & $P$ & NQ & PQ & $\mathrm{CQ}$ & $P$ \\
\hline & $\mathrm{n}=7$ & $\mathrm{n}=2$ & $\mathrm{n}=6$ & & $\mathrm{n}=6$ & $\mathrm{n}=3$ & $\mathrm{n}=6$ & \\
\hline Densidad aparente $\left(\mathrm{g} \mathrm{cm}^{-3}\right)$ & $0,50 \pm 0,05$ & $0,70 \pm 0,15$ & $0,60 \pm 0,06$ & 0,3167 & $0,96 \pm 0,04$ & $0,97 \pm 0,07$ & $0,97 \pm 0,06$ & 0,9948 \\
\hline $\mathrm{pH}$ agua & $6,7 \mathrm{a} \pm 0,1$ & $7,3 b \pm 0,1$ & $7,2 b \pm 0,1$ & 0,0005 & $6,8 \pm 0,1$ & $7,0 \pm 0,3$ & $6,4 \pm 0,1$ & 0,0772 \\
\hline Conductividad ( $\left.\mathrm{dS} \mathrm{m}^{-1}\right)$ & $0,06 \mathrm{a} \pm 0,00$ & $0,16 b \pm 0,02$ & $0,14 b \pm 0,02$ & 0,0009 & $0,05 \pm 0,01$ & $0,07 \pm 0,01$ & $0,06 \pm 0,01$ & 0,3255 \\
\hline $\mathrm{N}\left(\mathrm{g} \mathrm{kg}^{-1}\right)$ & $4,4 \mathrm{a} \pm 0,2$ & $3,7 \mathrm{ab} \pm 0,0$ & $3,3 b \pm 0,2$ & 0,0014 & $3,3 \pm 0,2$ & $4,0 \pm 0,3$ & $4,8 \pm 0,7$ & 0,1489 \\
\hline $\mathrm{C}\left(\mathrm{g} \mathrm{kg}^{-1}\right)$ & $74,2 \mathrm{a} \pm 3,0$ & $51,0 \mathrm{~b} \pm 5,0$ & $43,6 b \pm 2,8$ & 0,0001 & $49,7 \pm 4,0$ & $59,6 \pm 5,2$ & $70,2 \pm 9,9$ & 0,2758 \\
\hline $\mathrm{P}\left(\mathrm{mg} \mathrm{kg}^{-1}\right)$ & $29,4 \pm 5,1$ & $32,2 \pm 10,7$ & $17,4 \pm 3,4$ & 0,1177 & $20,6 \pm 3,9$ & $20,0 \pm 2,1$ & $20,9 \pm 2,0$ & 0,9124 \\
\hline $\mathrm{Ca}\left(\mathrm{cmol}+\mathrm{kg}^{-1}\right)$ & $21,6 \pm 1,6$ & $23,4 \pm 0,3$ & $20,1 \pm 0,7$ & 0,6336 & $18,5 \pm 1,5$ & $25,6 \pm 2,1$ & $24,5 \pm 2,3$ & 0,0608 \\
\hline $\mathrm{Mg}\left(\mathrm{cmol}+\mathrm{kg}^{-1}\right)$ & $2,9 \mathrm{a} \pm 0,2$ & $2,0 \mathrm{~b} \pm 0,0$ & $2,1 b \pm 0,2$ & 0,0388 & $2,5 \mathrm{a} \pm 0,2$ & $4,7 \mathrm{ab} \pm 1,3$ & $5,6 \mathrm{~b} \pm 0,5$ & 0,0028 \\
\hline $\mathrm{K}\left(\mathrm{cmol}+\mathrm{kg}^{-1}\right)$ & $0,90 \pm 0,07$ & $0,88 \pm 0,24$ & $0,97 \pm 0,19$ & 0,9891 & $1,57 \pm 0,14$ & $1,88 \pm 0,26$ & $2,65 \pm 0,74$ & 0,3137 \\
\hline $\begin{array}{l}\mathrm{N} \text { en biomasa microbiana } \\
\left(\mathrm{mg} \mathrm{kg}^{-1}\right)\end{array}$ & $61,4 \mathrm{a} \pm 5,4$ & $11,0 \mathrm{~b} \pm 4,0$ & $4,2 \mathrm{c} \pm 0,8$ & 0,0001 & $44,8 \pm 5,6$ & $41,3 \pm 1,9$ & $58,7 \pm 6,3$ & 0,1388 \\
\hline
\end{tabular}

NQ: no quemado; PQ: parcialmente quemado, CQ: completamente quemado; n: unidades muestrales por tratamiento. Las medias con letras distintas son diferentes $(P<0,05)$.

Treatments were: unburned (NQ), partially burned (PQ) and completely burned (CQ) forest. n: sampling units per treatment. Means with different letters were different $(P<0.05)$ 
tratamientos (cuadro 1). Salvo el nitrógeno, el resto de las variables que se diferenciaron significativamente del área no afectada no presentaron diferencias en las dos categorías de severidad (moderada y fuerte).

En Trevelin sólo se detectó mayor cantidad de magnesio en el tratamiento $\mathrm{CQ}$ que en el NQ, mientras que el PQ presentó valores intermedios $(P<0,05)$. En el resto de las variables las diferencias no fueron significativas $(P>0,05)$, detectándose una leve tendencia de aumento del calcio en los tratamientos quemados $(P=0,06)$ (cuadro 1 ).

\section{DISCUSIÓN}

En El Bolsón, a menos de dos años del fuego, se detectaron cambios en algunas propiedades del suelo relacionados principalmente a la combustión de la materia orgánica del suelo, mientras que en Trevelin luego de cinco años del fuego los cambios ocurridos en el suelo parecen haberse recuperado. La recuperación de la materia orgánica comienza con la recolonización de la vegetación que en general es rápida (Raison 1979) tal como se registró en este sitio (Urretavizcaya et al. 2006). Esta recuperación que se relaciona, en general, con la alta productividad primaria neta de la sucesión secundaria (Certini 2005) está asociada a los nutrientes que retornan al suelo en las cenizas y a una complejidad de factores (Raison 1979).

En general, las propiedades físicas de los suelos son menos afectadas por el fuego que las químicas, pero en el caso de que fuesen afectadas, éstas se recuperan más lentamente (Agee 1993). Al igual que en otros lugares (Moehring et al. 1966, Goh y Phillips 1991), en este estudio la densidad aparente no fue afectada por el fuego en ninguno de los dos sitios. El aumento en la alcalinidad de la capa superficial del suelo y en la conductividad eléctrica en El Bolsón se explica porque, al quemarse la materia orgánica, las sustancias minerales son liberadas en forma de óxidos o carbonatos que usualmente tienen reacción alcalina, aumentando la concentración de sales del suelo (Kutiel et al. 1990, Andreu et al. 1996). La magnitud y duración del cambio depende del pH original y contenido de materia orgánica del suelo, la cantidad y composición química de la ceniza así como de la precipitación local (Black 1975, Pritchett 1986, Chandler et al. 1991), pero en general disminuye con el tiempo (Iglesias et al. 1997). La disminución del carbono y nitrógeno en las zonas quemadas se produce por la combustión del mantillo y materia orgánica del suelo. Dado que los umbrales de temperatura del carbono y el nitrógeno son bajos en relación a las temperaturas que alcanzan los combustibles de madera, estos nutrientes son rápidamente volatilizados de la materia orgánica durante la combustión (Dyrness et al. 1989, DeBano 1990, Giovannini et al. 1990, Gillon et al. 1995, O'Connell y McCaw 1997). La disminución del carbono total del suelo luego de dos años del fuego se ha indicado en distintos estudios y se generaliza para los distintos componentes del carbono del suelo: carbono orgánico, carbono extraíble, carbono soluble en agua, ácidos húmicos y carbohidratos (Knoepp et al. 2005).

Existe una controversia en cuanto a la alteración de la concentración del nitrógeno en el suelo a causa del fuego, ya que se han reportado trabajos de incremento, disminución y de no modificación. Como regla general, tal cual lo observado en El Bolsón, la cantidad de nitrógeno que es volatilizado durante la combustión es directamente proporcional a la cantidad de materia orgánica consumida. El nitrógeno que no es completamente volatilizado permanece como parte de los combustibles no quemados o es convertido a la forma más disponible como $\mathrm{NH}_{4}-\mathrm{N}$, permaneciendo en el suelo (Certini 2005). Este incremento temporal de nitrógeno disponible luego del fuego es utilizado rápidamente por las plantas dentro de los primeros años después de éste (Knoepp et al. 2005). La respuesta entonces de los diferentes ecosistemas a los cambios de nitrógeno varían con el tipo de vegetación, la fertilidad del suelo y la habilidad para reponerlo (Wan et al. 2001).

El destino de la materia orgánica y el nitrógeno es de gran interés debido a su efecto positivo en las propiedades físicas del suelo y la liberación de nutrientes. El cambio en la materia orgánica y el nitrógeno está relacionado directamente con la magnitud de calentamiento del suelo y la severidad del fuego. Altas y moderadas severidades de fuego causan las mayores pérdidas en estos componentes tal como se observa en El Bolsón (Knoepp et al. 2005). Sin embargo, trabajos realizados en distintos ecosistemas (Weston y Attiwil 1990, Grogan et al. 2000) indican que pocos años después del fuego las cantidades de carbono y nitrógeno se recuperan a los valores previos a éste, tal como lo ocurrido en Trevelin. Esta recuperación se relacionaría con la descomposición de las raíces finas y la hojarasca de la vegetación que colonizó el área rápidamente (Kennard y Gholz 2001). En el largo plazo, los contenidos de carbono y nitrógeno tanto en los horizontes A como en las capas de suelo mineral cambian sólo en pequeñas cantidades (menos del 10\%) (Certini 2005).

Los cambios que produce el fuego en el ciclo de nutrientes como el magnesio son generalmente más leves y efímeros que los del nitrógeno. En general, si bien se reconoce que la disponibilidad del magnesio aumenta inmediatamente después del fuego (Knoepp et al. 2005), a menos de tres meses ese incremento puede desaparecer. Esta disminución temprana de magnesio está asociada a la mayor rapidez con que se lava este catión del suelo (Soto y Díaz-Fierros 1993). Una vez liberados por la combustión de la materia orgánica el magnesio y el calcio quedan como óxidos solubles fácilmente desplazados por el viento y por la escorrentía superficial, pero luego son rápidamente convertidos a carbonatos menos solubles y es por esa razón que estos cationes muestran usualmente altas retenciones en el suelo (Thiffault et al. 2008). Por ejemplo, el magnesio que se libera cuando se quema Eucalyptus spp. cambia a la forma insoluble dentro de los cuatro años por lo que 
las concentraciones de magnesio y calcio se incrementan considerablemente en la solución del suelo luego de un tiempo del fuego (Ludwig et al. 1998). Esto coincide con lo observado en Trevelin, así como por otros autores en bosques subboreales de Canadá y de Nothofagus spp. de Nueva Zelanda (Goh y Phillips 1991, Arocena y Opio 2003). Se indica en estos estudios que inmediatamente después del fuego hay una pequeña disminución de la cantidad de magnesio y calcio en la superficie del suelo mineral (Soto y Díaz-Fierros 1993), pero que luego hay un posterior incremento que ocurre simultáneamente con un aumento del fósforo. La razón de esta simultaneidad se debería a la formación de un fosfato de magnesio poco soluble y menos transportable en la mayoría de los suelos neutros (Viro 1974).

La inmovilización del nitrógeno en la biomasa microbiana en conjunto con su absorción por las plantas representan, a nivel de ecosistema, las vías de conservación más importantes del nitrógeno inorgánico del suelo (Vitousek 1981). El efecto del fuego sobre los microorganismos del suelo es substancial en la capa superficial, donde las poblaciones son abundantes y el efecto del calor es mayor (Valette et al. 1994). Sin embargo, va a depender del tipo de microorganismo y de la intensidad del evento, si el calentamiento del suelo es letal o solamente altera sus capacidades reproductivas (Neary et al. 1999). En El Bolsón, a 21 meses del evento de fuego, se observó una disminución significativa de N-BM asociada a la severidad de éste ( $84 \%$ y $93 \%$ menos en los tratamientos PQ y CQ en relación al testigo). Esta disminución en N-BM también fue observada en bosques quemados de Nothofagus pumilio (Poepp. et Endl.) Krasser (lenga) de la región andino patagónica de Argentina (Alauzis et al. 2004), donde se la encontró asociada a la pérdida de carbono orgánico, siendo éste el que regularía el N-BM (Mazzarino 2002). Aparte del efecto directo y letal del calentamiento del suelo, los cambios en la química del suelo pueden limitar el crecimiento de los microorganismos por cierto tiempo luego del fuego (Raison 1979). Si bien se han observado disminuciones de hasta el $96 \%$ en biomasa microbiana inmediatamente después de un evento de este tipo (Hernández et al. 1997), ningún ecosistema se mantiene estéril, aun después de un disturbio severo (Busse y DeBano 2005). Al igual que en Trevelin, distintos estudios muestran una recuperación de las poblaciones de organismos en el suelo mineral, a niveles de prefuego dentro de uno a cuatro años después del mismo (Vázquez et al. 1993, Prieto-Fernández et al. 1998). Las condiciones del sitio y las condiciones climáticas anteriores y posteriores al fuego son importantes, ya que pueden influenciar la naturaleza de éste y la recuperación del sitio (Neary et al. 1999). La velocidad de recuperación de las poblaciones de microorganismos está relacionada con el aumento de la temperatura del suelo, con la disponibilidad de nutrientes y humedad que favorezcan su crecimiento (Wright y Bailey 1982, Agee 1993, Busse y DeBano 2005).
Cambios postfuego en el suelo de bosques de Nothofagus spp. en la región andino patagónica (Alauzis et al. 2004) y de otras regiones (Kutiel et al. 1990, Andreu et al. 1996, Arocena y Opio 2003), presentaron tendencias similares a las encontradas en los bosques de A. chilensis estudiados. Asimismo, y dado que en El Bolsón se registró entre el primer y tercer año después del fuego (un año antes y uno después de este estudio respectivamente) un aumento de la cobertura del sotobosque de 42 a $72 \%$ en el tratamiento PQ y de 35 a $90 \%$ en el CQ (Urretavizcaya et al. 2006), sería interesante conocer de qué manera evolucionaron luego de este estudio las propiedades en el suelo quemado en relación a la recuperación de la vegetación. En el bosque de Trevelin, luego de cinco años del incendio, los cambios en las propiedades que pudieron haber ocurrido debido a este evento son imperceptibles, lo que demuestra una rápida recuperación del sitio.

El fuego genera diferentes condiciones en el suelo, que pueden tener implicancias en la emergencia de plántulas (Kennard y Gholz 2001) como sucede en Pinus halepensis Mill. (Pausas et al. 2003). Por ejemplo, para bosques de lenga del noroeste de la Patagonia argentina afectados por fuegos intensos en pequeños parches, se ha sugerido que la productividad postfuego y la regeneración estarían relacionadas con los cambios edáficos, especialmente aquellos que afectan la dinámica del nitrógeno y el agua (Alauzis et al. 2004). No obstante, estudios posteriores en la misma área sugieren la desaparición del mismo con el paso del tiempo al no corroborar ese efecto tres años después del fuego (Kitzberger et al. 2005). En los lugares estudiados la buena respuesta de $A$. chilensis a la siembra y plantación en las áreas quemadas (Urretavizcaya 2005, 2006) indicaría que la regeneración en el corto plazo no estaría controlada solamente por los cambios de fertilidad del suelo, sino por una conjunción de factores como la disponibilidad de semillas y los cambios en los patrones de temperatura de suelo en la áreas quemadas, asociados a las modificaciones de la cobertura arbórea y arbustiva luego del incendio (Urretavizcaya et al. 2006), factores que coinciden con los reportados Kitzberger et al. (2005) para la lenga.

Los resultados de este trabajo pueden aportan a las recomendaciones respecto al manejo postfuego de los bosques de A. chilensis. Es ampliamente conocido que los mayores riesgos de erosión postfuego ocurren en la estación de lluvia inmediatamente después del disturbio (Goh y Phillips 1991, DeBano 2000, Robichaud et al. 2003, Neary et al. 2005, Cerdá y Doerr 2005). También es sabido que la extracción de la madera puede aumentar el riesgo de erosión y la pérdida de nutrientes (Beschta et al. 1995, 2004, McIver y Starr 2000, Page-Dumroese et al. 2006). Asegurar que la vegetación postincendio se establezca para mitigar las posibles pérdidas de nutrientes y favorecer la recuperación de las propiedades de suelo, previamente a la extracción de la madera quemada puede ser, junto con la planificación de la restauración por plantación, una medida a implementar en el manejo de estas áreas. 


\section{AGRADECIMIENTOS}

Deseo agradecer a Luciano Taladriz por su ayuda en los trabajos de campo. A Alina Greslebin y Héctor Gonda por sus comentarios sobre el manuscrito. A Patricia Satti y a los dos revisores anónimos cuyas sugerencias ayudaron a mejorar el manuscrito. Este trabajo fue financiado por la Fundación Internacional para la Ciencia (IFS), Estocolmo, Suecia, a través del subsidio D/3120-1 y por la Comisión Nacional de Ciencia y Técnica de Argentina (CONICET) mediante una beca de formación de postgrado.

\section{REFERENCIAS}

Agee JK. 1993. Fire ecology on Pacific Northwest. Washington, D.C. USA. Island Press. 493 p.

Alauzis V, MJ Mazzarino, E Rafaelle, L Roselli. 2004. Wildfire in NW Patagonia: long-term effects on a Nothofagus forest soil. Forest Ecology and Management 192: 131-142.

Arbuinés MR. 1998. Relevamiento y estudio del régimen climático de la provincia del Chubut. Informe del plan de trabajo $\mathrm{N}^{\circ}$ 1020. Estación Experimental Agropecuaria Chubut. Trelew, Argentina. INTA.

Andreu V, JL Rubio, J Corteza, R Cerni. 1996. Postfire effects on soil properties and nutrients losses. International Journal of Wildland Fire 6: 53-58.

Arocena JM, C Opio. 2003. Prescribed fire-induced changes in properties of sub-boreal forest soils. Geoderma 113:1-16.

Baker DL, FS Guthery. 1990. Effects of continuous grazing on habitat and density of ground-foraging birds in south Texas. Journal of Range Management 43(1):2-5.

Beschta RL, CA Frissell, R Gresswell, R Hauer, J Karr, GW Minshall, DA Perry, JJ Rhodes. 1995. Wildfire and salvage logging. Recommendations for Ecologically Sound. PostFire Salvage Management and Other Post-Fire Treatments On Federal Lands in the West. 16 p.

Beschta RL, JJ Rhodes, JB Kauffman, RE Gresswell, GW Minshall, JR Karr, DA Parry, ER Hauer, CA Frissell. 2004. Postfire Management on Forested Public Lands of the Western United States. Conservation Biology 38: 957-967.

Black CA. 1975. Relaciones suelo-planta. Tomo I. Ed. Hemisferio Sur, Argentina. 866 p.

Bornemisza E. 1982. Introducción a la química de suelos. Secretaría General de la Organización de los Estados Americanos. Programa Regional de Desarrollo Científico y Tecnológico. Serie de Química, Monografía No 25.74 p.

Bran D, A Pérez, D Barrios, M Pastorino, J Ayesa. 2002. Ecoregión valdiviana: distribución actual de los bosques de "ciprés de la cordillera" (Austrocedrus chilensis) - Escala 1:250.000. INTA, APN, FVSA. Informe preliminar. Bariloche, Argentina. $12 \mathrm{p}$.

Buamscha G, M Gobbi, MJ Mazzarino, F Laos. 1998. Indicators of nitrogen conservation in Austrocedrus chilensis forests along a moisture gradient in Argentina. Forest Ecology and Management 112: 253-261.

Bustos JC, VC Rocchi. 1993. Caracterización termo-pluviométrica de veinte estaciones meteorológicas de Río Negro y Neuquén. Informe Técnico INTA EEA Bariloche. Bariloche, Argentina. 43 p.
Busse MD, LF DeBano. 2005. Soil Biology. In Neary DG, KC Ryan, LF DeBano eds. Wildland fire in ecosystems. Effects of fire on soil and water. Gen. Tech. Rep. RMRS-GTR42-vol. 4 (revised 2008). USDA, Forests Service, Rocky Mountain Research Station. p. 73-91.

Cerdá A, SH Doerr. 2005. The influence of vegetation recovery on soil hydrology and erodibility following fire: an elevenyear investigation. International Journal of Wildland Fire 14(4): 423-437.

Certini G. 2005. Effects of fire on properties of forest soils: a review. Oecologia 143: 1-10.

Chandler C, P Cheney, L Trabaud, D Williams. 1991. Fire in Forestry. Volumen I. Forest fire behavior and effects. Florida, Krieger Publishing Company. 450 p.

Christensen NL. 1987. The biogeochemical consequences of fire and their effects on the vegetation of the Coastal Plain of south-eastern United States. In Trabaud L. ed. The role of fire in ecological systems, SPB Academic Publishing. p. 1-19.

Colmet Dâage F, ML Lanciotti, AA Marcolin. 1995. Importancia forestal de los suelos volcánicos de la Patagonia Norte y Central. Climo-topo-secuencia de suelos y vegetación. Centro Regional Patagonia Norte, EEA Bariloche. 27 p.

Colmet Dâage F, MJ Mazzarino, AA Lanciotti. 1993. Características de los suelos volcánicos en el S.O. del Chubut. Comunicación Técnica $\mathrm{N}^{\circ}$ 22. Recursos Naturales-Suelos. INTA. EEA Bariloche. 10 p.

Connell JH, RO Slatyer. 1977. Mechanisms of succession in natural communities and their role in community stability and organization. American Naturalist 111: 1119-1144.

DeBano LF. 1990. The effect of fire on soil properties. Symposium on Management and Productivity of Western-Montane Forest Soil, Boise, Idaho. April 10-12.

DeBano LF. 2000. The role of fire and soil heating on water repellency in wildland environments: a review. Journal of Hydrology 231-232: 195-206.

DeBano LF, DG Neary, PF Ffolliott. 1998. Fire's effects on ecosystems. New York, USA. John Wiley. 333 p.

Díaz-Fierros F, E Benito, JA Vega, A Castelão, B Soto, R Pérez, T Taboada. 1990. Solute loss and soil erosion in burnt soil from Galicia (NW Spain). In Goldammer JG, MJ Jenkins eds. Fire and Ecosystem Dynamics. SPB Academic Publishing, The Netherlands. p. 103-116.

Donoso C. 1981. Ecología forestal. El bosque y su medio ambiente. Editorial Universitaria. Universidad Austral de Chile. Santiago de Chile. 369 p.

Donoso C. 1993. Bosques templados de Chile y Argentina. Editorial Universitaria. Universidad Austral de Chile. Santiago de Chile. 484 p.

Dormaar JF, S Smoliak, WD Willms. 1990. Distribution of nitrogen fractions in grazed and ungrazed fescue grassland Ah horizons. Journal of Range Management 43(1): 6-9.

Dunn PH, LF DeBano. 1977. Fire's effect on biological and chemical properties of chaparral soils. Proceedings of a Symposium on Environmental Conservation: Fire and Fuel Management in Mediterranean Ecosystems. August 4-5, 1988. Palo Alto, C.A. Washington, D.C. USDA For. Serv. WO-3. p 75-84.

Dyrness CT, K Van Cleve, JD Levison. 1989. The effect of wildfire on soil chemistry in four forest types in interior Alaska. Canadian Journal of Forest Research 19(11): 1389-1396. 
Etchevehere PH. 1972. Los suelos de la Región Andino Patagónica. In Dimitri MJ ed. La Región de los Bosques AndinoPatagónicos, Sinopsis General. Colección Científica del INTA. Buenos Aires, Argentina. p. 83-95.

Gillon D, V Gomendy, C Houssard, J Maréchal, J Valette. 1995. Combustion and nutrient losses during laboratory burns. International Journal of Wildland Fire 5(1):1-12.

Giovannini G, S Luchessi, M Giacheti. 1990. Beneficial and detrimental effects of heating on soil quality. In Goldammer JG, MJ Jenkins eds. Fire and Ecosystem Dynamics. SPB Academic Publishing, The Netherlands. p. 95-102.

Goh KM, MJ Phillips. 1991. Effects of clearfell logging and clearfell logging and burning of a Nothofagus forest on soil nutrient dynamics in South Island, New Zealand- changes in forest floor organic matter and nutrient status. New Zealand Journal of Botany 29: 367-384.

Grogan P, TD Bruns, FS Chapin. 2000. Fire effects on ecosystem nitrogen cycling in Californian bishop pine forest. Oecologia 122: $537-544$.

Hernández T, C. García, I Reinhardt. 1997. Short-term effect of wildfire on the chemical, biochemical, and microbiological properties of Mediterranean pine forest soils. Biology and Fertility of Soils 25: 109-116.

Hurlbert SH. 1984. Pseudoreplication and the design of ecological field experiments. Ecological Monographs 54: 187-211.

Iglesias MT, V Cala, J Gonzáles, I Walter, L Trabaud. 1997. Fire effects during two years on soil nutrients in a Juniperus oxycedrus woodland. In Trabaud L ed. Fire Management and Landscape Ecology. International Association of Wildland Fire, Fairfield, WA. p. 13-24.

Irisarri J. 2000. La propuesta de reclasificación de los Andepts de Argentina, de acuerdo al Orden Andisoles. Actas Workshop Soil Taxonomy, Instituto Nacional de Tecnología Agropecuaria, AICET, Asociación Argentina de la Ciencia del Suelo. p. 18-27.

Kennard DK, HL Gholz. 2001. Effects of high- and low-intensity fires on soil properties and plant growth in a Bolivian dry forest. Plant and Soil 234: 119-129.

Kitzberger T. 1994. Fire regime variation along a northern Patagonian forest-steppe-ecotone: stand a landscape response. Tesis de Doctorado. Department of Geography, University of Colorado, Boulder, Colorado. 203 p.

Kitzberger T, E Raffaele, K Heinemann, MJ Mazzarino. 2005. Effects of fire severity in a north Patagonian subalpine forest. Journal of Vegetation Science 16: 5-12.

Knoepp JD, LF DeBano, DG Neary. 2005. Soil Chemistry. In Neary DG, KC Ryan, LF DeBano eds. Wildland fire in ecosystems. Effects of fire on soil and water. Gen. Tech. Rep. RMRS-GTR-42-vol.4 (revised 2008). USDA, Forests Service, Rocky Mountain Research Station. p. 53-71.

Kutiel P, Z Naveh, H Kutiel. 1990. The effect of a wildfire on soil nutrients and vegetation in an aleppo pine forest on Mount Carmel, Israel. In Goldammer JG, MJ Jenkins eds. Fire and Ecosystem Dynamics. The Netherlands. SPB Academic Publishing. p.85-94.

La Manna L. 2005. Caracterización de los suelos bajo bosque de Austrocedrus chilensis a través de un gradiente climático y topográfico en Chubut, Argentina. Bosque 26(2): 137-153.

López C, F Collmet Dâage, H Planas. 1992. Características de los suelos de origen volcánico en la cordillera Andino- patagónica Latitud $43^{\circ} 30^{\prime}$ S. Argentina-Chile. Comunicación Técnica $N^{\circ}$ 18. Recursos Naturales-Suelos. INTA. EEA Bariloche. 15 p.

Ludwig B, PK Khanna, RJ Raison, KL Jacobsen. 1998. Modelling cation composition of soil extracts under ashbeds following an intense slashfire in a eucalypt forest. Forest Ecology and Management 103: 9-20.

McIver JD, L Starr. 2000. Environmental effects of postfire logging: literature review and annotated bibliography. General Technical Report PNW-GTR-486. Portland, Oregon, USA. USDA Forest Service. Pacific Northwest Research Station. $74 \mathrm{p}$.

Mutch LS, TW Swetnam. 1995. Effects of fire severity and climate on Ring-Width Growth of Giant Sequoia after Burning. In Brown JK, RW Mutch, CW Spoon, RH Wakimoto eds. Proceedings: Symposium on fire in wilderness and park management. Missoula, March 30 - April 01, 1993. Report INT-GTR-320. Missoula, USA. USDA Forest Service. Intermountain Research Station. p. 241-246

Mazzarino MJ. 2002. Circulación de Nutrientes en Ecosistemas Naturales: Conservación en la Vegetación y Dinámica en el Suelo. In Actas XVIII Congreso Argentino de la Ciencia del Suelo. Puerto Madryn, Argentina.

Moehring DM, CX Grano, JR Bassett. 1966. Properties of forested loess soils after repeated prescribed burns. Research Note. New Orleans, LA, USA. US, Forest Service, SFES. 4 p.

Murphy J, JP Riley. 1962. A modified single solution method for the determination of phosphate in natural waters. Analytica Chimica Acta 27: 31-36

Neary DG, CC Klopatek, LF DeBano, PF Ffolliott. 1999. Fire effects on belowground sustainability: a review and synthesis. Forest Ecology and Management 122: 51-71.

Neary DG, KC Ryan, LF DeBano, JD Landsberg, JK Bronw. 2005. Introduction. In Neary DG, KC Ryan, LF DeBano eds. Wildland fire in ecosystems. Effects of fire on soil and water. Gen. Tech. Rep. RMRS-GTR-42-vol.4 (revised 2008). USDA, Forests Service, Rocky Mountain Research Station. p. 1-17.

O'Connell AM, WL McCaw. 1997. Prescribed burning of thinning slash in regrowth stand of karri (Eucalyptus diversicolor) 2 . Nitrogen budgets in pre- and post-burn fuel. International Journal of Wildland Fire 7(1): 41-50.

Page AL, RH Miller, DR Keeney. 1982. Methods of soil analysis. Part 2. Chemical and microbiological properties. $2^{\text {nd }}$ ed. Madison, Wisconsin, USA. American Society of Agronomy. $1159 \mathrm{p}$.

Page-Dumroese D, M Jurgensen, A Abbott, T Rice, J Tirocke, S Farley, S DeHart. 2006. Monitoring changes in soil quality from post-fire logging in the Inland Northwest. USDA Forest Service Proceedings RMRS-P-41. p 605-614.

Pastorino MJ, LA Gallo 2000. Variación geográfica en peso de semilla en poblaciones naturales argentinas de "Ciprés de la Cordillera”. Bosque 21(2): 95-109.

Pausas JG, N Ouadah, A Ferran, T Gimeno, R Vallejo. 2003. Fire severity and seedling establishment in Pinus halepensis woodlans, Iberian Peninsula. Plant Ecology 169: 205-213.

Prieto-Fernández A, MJ Acea, T Carballas. 1998. Soil microbial and extractable $\mathrm{C}$ and $\mathrm{N}$ after wildfire. Biology and Fertility of Soils 27: 132-142.

Pritchett WL. 1986. Suelos Forestales. Propiedades, Conservación y Mejoramiento. México. Limusa. 634 p. 
Pyne SJ. 1984. Introduction to Wildland Fire. Fire Management in the United States. New York, USA. John Wiley. 455 p.

Raison RJ. 1979. Modification of the soil environmental by vegetation fires, with particular reference to nitrogen transformations: a review. Plant and Soil 51:73-108.

Robichaud P, L MacDonald, J Freeouf, D Neary, D Martin, L Ashmun. 2003. Postfire Rehabilitation of the Hayman Fire. USDA Forest Service Gen. Tech. Rep. RMRSGTR-114. 293 p.

SAS. 1999. Manual of software versión 8 (TS MO), SAS Institute Inc. Cary, NC, USA.

Schlegel F. 1962. Hallazgo de un bosque de cipreses cordilleranos en la provincia de Aconcagua. Boletín de la Universidad de Chile 32: 43-46.

Singh RS. 1994. Changes in soil nutrients following burning on dry tropical Savanna. International Journal of Wildland Fire 4(3):187-194.

Sokal RR, FJ Rohlf. 1981. Biometry: the principles and practice of statistics in biological research. Freeman, San Francisco, CA. 362 p.

Soto B, F Diaz-Fierros. 1993. Interactions between plant ash leachates and soil. International Journal of Wildland Fire 3(4): 207-216

Sparks DL, AL Page, PA Helmke, RH Loeppert, PN Soltanpour, MA Tabatabai, CT Johnston, ME Sumner. 1996. Methods of Soil Analysis. Part 3. Chemical Methods. SSSA Book Series Nr. 5. Madison, Wisconsin, USA. SSSA, ASA. p. $437-474$.

Swindale L. 1969. Propiedades de los suelos de cenizas volcánicas. Panel sobre suelos derivados de ceniza volcánica de América Latina. Turrialba, Costa Rica. IICA. 9 p.

Thiffault E, KD Hannam, SA Quideau, D Paré, N Bélanger, SW Oh, AD Munson. 2008. Chemical composition of forest floor and consequences for nutrient availability after wildfire and harvesting in the boreal forest. Plant Soil 308: 37-53.

Thurow TL, WH Blackburn, CA Taylor. 1988. Some vegetation responses to selected livestock grazing strategies, Edwards Plateau, Texas. Journal of Range Management 41(2): $108-114$

Trabaud L, C Michels, J Grosman. 1985. Recovery of burnt Pinus halepensis Mill. Forests. I. understorey and litter phytomass development after wildfire. Forest Ecology and Management 13: 269-277.

Urretavizcaya MF. 2005. Cambios ambientales y restauración ecológica postincendio en bosques de Austrocedrus chilensis. Tesis de Doctorado. Bariloche, Argentina. Universidad Nacional del Comahue. 205 p.

Urretavizcaya MF. 2006. Restauración postfuego de bosques de ciprés de la cordillera: comportamiento de distintos plantines a 4 y 5 años de su plantación. In Segunda Reunión Patagónica y Tercera Nacional sobre Manejo y Ecología del Fuego - Eco-Fuego 2006. Esquel, abril de 2006. CIEFAPUNPat-PNMF-DGByP.

Urretavizcaya MF, GE Defossé. 2004. Soil seed bank of Austrocedrus chilensis (D. Don) Pic. Serm. et Bizarri related to different degrees of fire disturbance in two sites of southern Patagonia, Argentina. Forest Ecology and Management 187: 361-372.
Urretavizcaya MF, GE Defossé, HE Gonda. 2006. Short-term effects of fire on plant cover and soil conditions in two Austrocedrus chilensis (cypress) forests in Patagonia, Argentina. Annals of Forest Science 63: 63-71.

Valette JC, V Gomendy, J Maréchal, C Houssard, D Gillon. 1994. Heat transfer in the soil during very low-intensity experimental fire: the role of duff and soil moisture content. International Journal of Wildland Fire 4(4): 225-237.

Vázquez FJ, MJ Acea, T Carballas. 1993. Soil microbial populations after wildfire. Microbial Ecology 13: 93-104.

Veblen TT, T Kitzberger, A Lara. 1992. Disturbance and forest dynamics along a transect from Andean rain forest to Patagonian shrubland. Journal of Vegetation Science 3: 507-520.

Veblen TT, T Kitzberger, BR Burns, AJ Rebertus. 1996. Perturbaciones y dinámica de regeneración en bosques andinos del sur de Chile y Argentina. In Armesto JJ, C Villagrán, MK Arroyo eds. Ecología de los bosques nativos de Chile. Santiago, Chile. Editorial Universitaria. p. 169-198.

Veblen TT, B Burns, T Kitzberger, A Lara, R Villalba. 1995. The Ecology of the Conifers of Southern South America. In Enright N, R Hill eds. Ecology of the Southern Conifers. Melbourne University Press, Carlton, Victoria, Australia. p. 120-142.

Veblen TT, T Kitzberger, E Raffaele, M Mermoz, ME González, JS Sibold, A Holz. 2008. The historical range of variability of fires in the Andean-Patagonian Nothofagus forest region. International Journal of Wildland Fire 17(6): 724-741.

Viro PJ. 1974. Effects of forest fire on soil. In Kozlowski T, CE Ahlgren eds. Fire and Ecosystems. Academic Press, New York. p. 7-46.

Vitousek PM. 1981. Clear-cutting and the nitrogen cycle. In Clark FE, T Roswall eds. Terrestrial Nitrogen Cycles. Ecological Bulletin 33: 631-642.

Vitousek PM, PA Matson. 1985. Disturbance, N-availability and N-losses: An experimental study in an intensively managed loblolly pine plantation. Ecology 66: 1360-1376.

Walkley A, I A Black. 1934. An examination of Degtjareff method for determining soil organic matter and a proposed modification of the chromic acid titration method. Soil Sci. 37:29-37.

Wan S, D Hui, Y Luo. 2001. Fire effects on nitrogen pools and dynamics in terrestial ecosystems: a meta-analysis. Ecology 82(11): 1349-1365.

Wester DB. 1992. Viewpoint: replication, randomization, and statistics in range research. Journal of Range Management 45(3): 285-290.

Weston CJ, PM Attiwill. 1990. Effects of fire and harvesting on nitrogen transformations and ionic mobility in soil of Eucaliptus regnans forests of southeastern Australia. Oecologia 83: 20-26.

Williams RJ, RA Bradstock. 2008. Large fires and their ecological consequences: introduction to the special issue. International Journal of Wildland Fire 17(6): 685-687.

Wright HA, AW Bailey. 1982. Fire Ecology, United States and Southern Canada. New York, USA. John Wiley. 501 p.

Recibido: 27.07.09

Aceptado: 16.03.10 